\title{
The influence of achievement goal orien- tation on study time allocation
}

\author{
Hui Zheng ${ }^{1}$ Kaikai Chen ${ }^{1}$ Yuqing Lin $^{1}$ Huaqiang Tang ${ }^{1}$ Utete Fanuel E. ${ }^{1}$ \\ Xiaoyu $\mathrm{Jia}^{2} \mathrm{Xinyu} \mathrm{Li}^{2}$ Weijian $\mathrm{Li}^{2}$ \\ ${ }^{1}$ Department of Psychology, Zhejiang Normal University, Jinhua, 321004 \\ ${ }^{2}$ Institute of Psychology, Zhejiang Normal University, Jinhua, 321004
}

\begin{abstract}
The purpose of this study was to investigate the effect of different types of achievement goal orientation on learner's study time allocation. A 2(achievement goal orientation: mastery goal, performance orientation) $\times 2$ (item reward: 1 point, 5 point) $\times 2$ (item difficulty: easy item, hard item) mixed design was adopted. The results showed that the performance orientation learners tended to select and allocate more learning time to 5point items. However, the mastery goal oriented learners did not show such selection bias. According to the ABR framework, mastery goal orientation learner developed a more flexible agenda.
\end{abstract}

Keywords: Metacognition, Study Time Allocation, Achievement Goal Orientation

\section{Introduction}

Study time allocation is the management and control of psychological resources, which reflects one's understanding of the task and the ability of selective participation(Perfect \& Schwartz, 2002). It is a planned behaviour that learners allocate their time resources into different tasks ( $\mathrm{Li}, 2011$ ), which includes item selection and a self-paced learning process. (Son \& Sethi, 2006).
There are three main theoretical models to explain the internal mechanisms of study time allocation, which respectively refer to Discrepancy Reduction Model, Hierarchical Model, and Proximal Learning Model (RPL Model). The Discrepancy Reduction Model (Dunlosky \& Hertzog, 1998) predicts that novices unfamiliar with a task or stimuli will focus on unknown rather than known items and initially select and allocate more time to the least well known items in order to reduce the discrepancy between what is currently known and the desired level of knowledge. Then, to account for the extant data on self-paced study and item selection, Thiede and Dunlosky (1999) proposed a Hierarchical Model that consists of a "subordinate level in which the regulation of individual items occurs, along with a superordinate level that involves planning in which decisions about how to regulate study are made on the basis of how to complete the task as a whole". The idea is that an individual first plans how to regulate study across all items of a list, and following control is transferred to a subordinate system in which individual items are studied. In contrast, Metcalfe's (2002) Region of RPL Model presents that individuals will first select easier unknown items when study time is limited because these items are more likely to be within a range of items that people believe they can learn. However, these three theoretical models are mostly 
concerned with only one factor (i.e. the difficulty of items) influencing the allocation of time for learning. Actually study time is also affected by other factors such as item value (Dunlosky \& Thiede, 1998; Niuet'al, 2006). Ariel, Dunlosky and Bailey (2009) adopted the paradigm includes learning within a fixed time frame, Judgment of Learning (JOL), interference, first recall test, self-paced re-learning and subsequent recall tests, (the Dunlosky method), to explore, these two factors(the difficulty and values of items)' influences on the allocation of time for learning. It was found that item values surpass the item difficulty and produce a more significant impact on study-time allocation. Based on these results, Areal et'al (2009) proposed the Agenda Based Regulation Model (ABR Model). The model assumes that learners' resource management includes agenda allocation and framing. Learners develop an agenda based on the way of allocating time to various study items and use this agenda to decide when to select items for study. The model also suggests that decision level of study-time allocation is affected by other factors, such as metacognition, difficulty of item, value of item and other external factors, and it is also influenced by learners' achievement based motivation in the learning process, standards, decision making style, working memory, attention and other individual factors. However these hypotheses of ABR model need more empirical evidence to support. For instance, how the achievement goals affect the learners' agenda building process, and thus have an impact on study-time allocation? Above questions will be explored in this study.

As for the achievement goals, they can be divided into mastery goal orientation and performance goal orientation (Ames, 1988). The people who have a mastery goal orientation are inclined to pay more attention to the development of their abil- ities and have an evaluation for their efforts. They focus on understanding and mastering of a task. On the contrast, the people who are performance goal orientated pay more attention on their abilities to be evaluated by others and tend to prove their ability via their excellent performance in learning process. Some researchers believe that a mastery goal, as a positive motivation model, will lead to give the learner a positive emotional learning experience and learning behaviour. Whereas, a performance goal contains a more negative form of motivation (Pintrich, 1993; Kaplan, 1999; Wigfeld, 2002; Stipeck, 2004). In line with these views, Sitzmann \& Ely(2011) found that mastery goal oriented people were positively correlated with self-regulation based on a meta-analysis study. Taking into account of these two different manifestations of achievement goal orientation, we assume that in a situation which contains item difficulty and item value, While people who have the mastery goal orientation tend to choose difficult item for learning and allocate more time on them, people who have the performance goal orientation are inclined to choose the items which will give them higher marks and allocate more time on those items.

In sum, this study will assess the influence of different types of achievement goals on study time allocation. A $2 * 2 * 2$ mixed design was adopted. The independent variables are the achievement goal orientation (between-subjects factor expressed by A, which is divided into two levels: al-mastery goal orientation and a2performance goal orientation), item difficulty (whithin-subjects factor expressed by $\mathrm{B}$, which is divided into two levels: b1-difficult item b2-easy item) and item value (whithin-subjects factor expressed by $\mathrm{C}$, which is also divided into two levels: c1- 1 point item, c2- 5 point item). 


\section{Method}

\subsection{Participants}

Participants were undergraduates from Zhejiang Normal University in China $(n=124)$ including 82 female students and 42 male students. There was a financial incentive for completing the experiment. They were then grouped randomly to the Mastery Goal Orientation Group ( $n=61)$ and Performance Goal Orientation Group $(\mathrm{n}=63)$.

\subsection{Material}

The study material included 60 Chinese noun word pairs taken by $\mathrm{Li}$ (2011).According to the degree of association, these pairs were divided into 30 easy word pairs and 30 difficult word pairs. All word pairs were randomly assigned point 1 or point 5 (i.e. different values).

\subsection{Procedure}

The Dunlosky paradigm (Ariel, Dunlosky and Bailey 2009) was adopted in current study to explore the participants' study time allocation. Before the experiment, we applied different introductory phrase to stimulate participates' different achievement goals (Thiede \& Dunlosky, 1999). All participants completed their test alone at their assigned workstation, without the aid of other material.

\subsection{Data Collection and Processing}

The participants' overall recall scores were calculated by the accurate rate. The participants' reselection of first six preceding tasks referred to the ratio of the number of reselections of particular tasks of project in the total number of options. The total time of self-paced study refers to the actual average study time distributed across the certain whole project. The processing of data was analyzed through SPSS 17.0.

\section{Results}

\subsection{Final recall}

To examine the influence of achievement goal orientation, item difficulty and item score on recall accuracy rate during final recall, the analysis of variance (ANOVA) of repeated-measures was performed. The results showed that the main effect of Item Difficulty was significant, $F(1,122)=222.66, \quad p<.001, \quad \eta^{2}=0.65$. The Item Difficulty $\times$ Achievement Goal Orientation interaction was significant, $F(1,122)=35.02, p<.001, \eta^{2}=0.24$.

\subsection{The first six re-learn-selection}

To examine the influence of achievement goal orientation, item difficulty and item score on learners'item selection for the first six relearn items, ANOVA of repeated-measures was performed for the date (Figure 1). The results showed that the main effect of Item Difficulty was significant, $F(1,122)=101.13, p<.001, \eta^{2}=$ 0.45 . The main effect of Item Value was significant, $F(1,122)=22.50, p<.001, \eta^{2}=$ 0.15 .

The Item Difficulty $\times$ Item Value interaction was significant, $F(1,122)=8.44$, $p<.05, \quad \eta^{2}=0.07$. The Item Score $\times$ Achievement Goal Orientation interaction was significant, $F(1,122)=16.77, p<.001$, $\eta^{2}=0.12$. The results of further analysis of simple effect showed that participants who adopted a performance goal orientation chose more 5-point items $(M=33.07)$ than the 1-point items $(M=16.93)$. However, in the mastery goal orientation group, there was no significant differences between 5-point items and 1-point items ( $p>>05)$. 


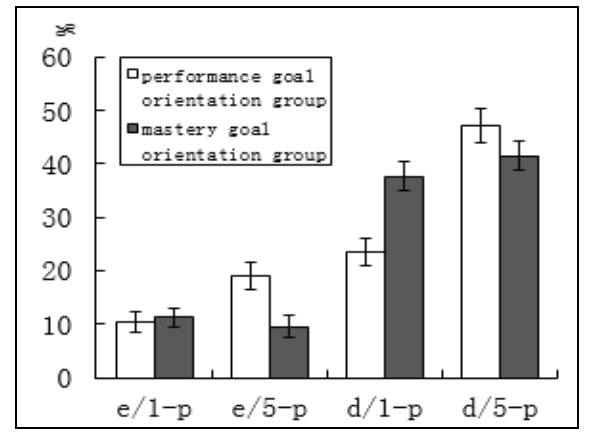

Fig. 1: Proportion of the first 6 items selected for restudy.

\subsection{Self-paced study time}

To examine the influence of achievement goal orientation, item difficulty and item score on time of self-paced study for all items, ANOVA of repeated-measures was presented for the study time which each participant spend on each task (i.e. easy-low value pairs, easy-high value pairs, hard-low value pairs or hard-high value pairs) (Figure 2). The results demonstrated that those issues are notable, which include the main effect of Item Difficulty was significant, $F(1,122)=11$ $0.31, p<.001, \eta^{2}=0.48$, and the Item Difficulty $\times$ Item Value interaction was significant, $F(1,122)=13.01, p<.001, \eta^{2}=0.97$. The Item Score $\times$ Achievement Goal Orientation interaction was significant, $F(1$, 122) $=5.37, p<.05, \eta^{2}=0.04$. Participants who adopted a performance goal orientation allocated more time to the 5-point items $(M=21.61)$ than the 1-point items $(M=15.85)$. The Interaction effects of Achievement Goal Orientation, Item Difficulty and Item Value was significant, $F(1,122)=5.88, p<.05, \eta^{2}=0.05$. The results of further analysis of simple effect showed that for the difficult items, participants who adopted a performance goal orientation allocated more time to the items of 5 score $(M=38.06)$ than the items of 1 score $(M=27.72)$. However, in the mastery goal orientation group, there was no significant difference between 5-point items and 1-point items ( $p$ s>.05).

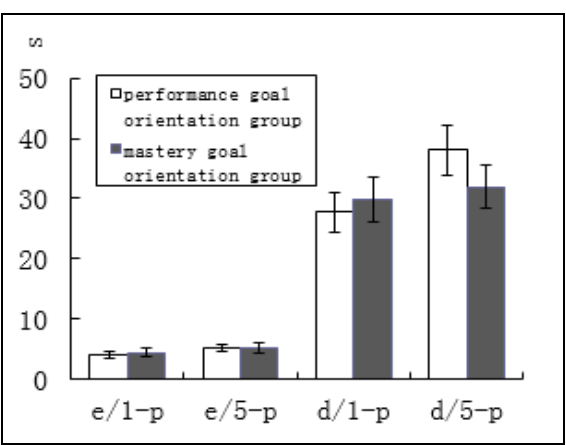

Fig. 2: Time of self-paced study for all items.

\section{Discussion}

There are three major findings of the current study. First, for easy items, the participants' final accuracy recall ratio in performance goal orientation group was significantly higher than those in mastery goal orientation group. Whereas, for hard items, the results were adverse. Secondly, during the first six re-learning step, the participants in performance goal orientation group were likely to choose 5-point items to re-learn. However, this effect was not found in mastery goal orientation group. Thirdly, in the self-paced learning time, for difficult items, the participants in performance goal orientation group allocated more study time to 5-point items. But the participants in mastery goal orientation group did not show this effect.

These results manifested that persons' achievement goal orientation affect their study time allocation process significantly, which provides empirical evidence for the "Agenda-based regulation model" (Ariel, Dunlosky, \& Bailey, 2009; Dunlosky \& Ariel, 2011). Based on the ABR model, "learners develop an agenda on how to allocate time to various study items and use this agenda when selecting 
items for study. Like other theories of regulation, the $\mathrm{ABR}$ model assumes that study regulation is goal-oriented "(Dunlosky \& Ariel, 2011).A critical assumption is that when the learners develop agendas, they try to efficiently accomplish their goals. Originally, the achievement goal orientation theory stressed two kinds of orientations to achievement: mastery and performance goals (Ames 1992; Dweck and Leggett 1998).Mastery goals oriented the student towards learning and understanding, developing new skills, and a focus on self-improvement to use self-referenced standards. Performance goals represent a concern with demonstrating ability, obtaining recognition of one's ability, protecting one's selfworth and a focus on comparative standards relative to surpass others (Pintrich 2000). Performance goal's negative motives are namely, aiming for a positive evaluation and the avoidance of a negative one at the expense of not fully grasping a concept. According to Ames (1992), mastery goal orientation learners will be more concerned about their capabilities, they always adjust the length of learning time according to their mastery of items and the importance of values is weakened or non-existent (Dweck \& Leggett, 1988). In line with these characteristics of persons with different achievement goal orientation, mastery goal orientation learners re-learned all non-mastered word pairs with an equal bias and the values of these re-learned items and had little effect on the choice to or not to re-learn a specific item. In contrast, the performance goal orientation learners' ultimate goal is to get high marks, so they paid more attention to high value items. When they found the items had different values, in order to maximize learning gains, they assigned more study time to the higher value items.

Li (2011) proposed a mechanism for study time allocation trade-off model.
This model is a trade-off study time allocation process, aiming at balancing an individual's cognitive dynamic processes. The internal factors, such as attention, working memory, achievement motivation, efficiency, interests, decisionmaking style, will affect individuals' agenda development. Consistent with the views of $\mathrm{Li}$, we found that achievement goal is an important individual factor which affects learners in formulating a study goal, thereby affecting learners' development and execution of an agenda. This finding enriches study-time allocation model and the internal mechanism of self-regulated learning theory.

\section{Discussion}

In the item selection and self-paced learning time, for different values we found that mastery goal orientated learners have no selection bias. The performance orientation learners tend to select and allocate more learning time in high value word-pairs.

This study was supported by the National Natural Science Foundation of China(No. 31170999) and the National Training Programs of Innovation and Entrepreneurship for Undergraduates.

Corresponding author: Xinyu $\mathrm{Li}$ (xyli@zjnu.cn); Weijian $\quad$ Li (xlxh@zjnu.cn).

\section{References}

[1] Ames, C. (1992). Classrooms: Goals, structures, and student motivation. Journal of educational psychology, 84(3), 261.

[2] Ariel, R., Dunlosky, J., \& Bailey, H. (2009). Agenda-based regulation of study-time allocation: When agendas override item-based monitoring. 
Journal of Experimental Psychology:

General, 138(3), 432.

[3] Coutinho, S. A., \& Neuman, G. (2008). A model of metacognition, achievement goal orientation, learning style and self-efficacy. Learning Environments Research, 11(2), 131151.

[4] Dunlosky, J., \& Thiede, K. W. (2004). Causes and constraints of the shift-toeasier-materials effect in the control of study. Memory \& Cognition, 32(5), 779-788.

[5] Murayama, K., \& Elliot, A. J. (2011). Achievement Motivation and
Memory Achievement Goals Differentially Influence Immediate and Delayed Remember-Know Recognition Memory. Personality and Social Psychology Bulletin, 37(10), 1339-1348.

[6] Sitzmann, T., \& Ely, K. (2011). A meta-analysis of self-regulated learning in work-related training and educational attainment: What we know and where we need to go. Psychological bulletin, 137(3), 421.

[7] Li W. (2011). Learning difficulties for children to learn the characteristics of time allocation. $\mathrm{PhD}$ thesis, Beijing Normal University. 